and released by the legendary Cornell Laboratory of Ornithology. MERLIN assists in bird identifications (allaboutbirds.org/guide/Merlin), and eBird allows birds' locations, numbers, and dates to be uploaded to global databases instantly (ebird.org). eBird can even compile lists of birds "likely" to be found at a specified spot. Perhaps the Winter Bird Atlas can be reborn in a smartphone?
REFERENCE

Reinking, Dan L., ed. 2004. Oklahoma Breeding Bird
Atlas. Norman, OK: University of Oklahoma Press.

\title{
SERVICE-ORIENTED MAPPING: CHANGING PARADIGM IN MAP PRODUCTION AND GEOINFORMATION MANAGEMENT
}

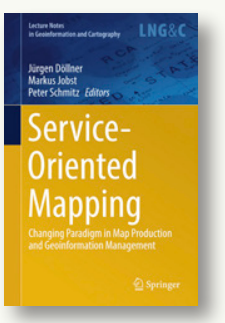

Edited by Jürgen Döllner, Markus Jobst, and Peter Schmitz

Springer, 2019

434 pages; \$189 hardcover, \$149 eBook.

ISBN: 978-3-319-72434-8

Review by: Amy Rock, Humboldt State University

The term "service-oriented mapping" refers here to mapping applications or map services, both Software-as-aService (SaaS) and Data-as-a-Service (DaaS), that in some way automate the delivery of map data to the intermediate or end user. In Service-Oriented Mapping: Changing Paradigm in Map Production and Geoinformation Management, the emphasis is largely on DaaS, and how to use it with desktop GIS products or free web-based interfaces. This book presents a generous collection of twenty service-oriented mapping examples from the international (predominantly European and African) community, and provides some insight into how national, multinational, and nongovernmental organizations are creating or leveraging these services to use or generate geographic data.

The chapters are organized into three parts: "Part I: Exploring a New Paradigm in Map Production," "Part II: Importance and Impact of the New Map Production Paradigm," and "Part III: Requirements of the New Map Production Paradigm." Part I leads off with a broad overview of issues related to the transition from paper to digital maps, emphasizing the evolution from long-lived artifacts presenting static, and eventually outdated, information, to more ephemeral electronic products involving interactive and potentially quickly updateable representations in keeping with the dynamic nature of many datasets. According to Service-Oriented Mapping, this new paradigm leverages the modern culture of "sharing and reuse" that requires us to think differently about modern map production. Other articles in this section discuss strategies for managing massive datasets and understanding their infrastructure needs, implementing custom applications, and developing automated processes to support on-demand mapping.

Part II, "Importance and Impact of the New Map Production Paradigm," provides a series of case studies and historical overviews of the transition to service-oriented mapping. While some of these articles are easily accessible to non-specialist audiences, others are highly technical, and include code samples and processes that are quite discipline-specific. Part III, "Requirements of the New Map Production Paradigm," is chiefly a discussion of the need for data and access standards to ensure interconnectivity and interoperability between various data systems and sources. It includes a study of how to define the needs of various user types to aid in developing standards - such as for metadata, storage formats, and accessibility - for archival entities.

It is clear the editors intended to present a range of service-oriented mapping applications, but the intended audience for their collection is less clear. The articles range in tone from a highly accessible white-paper style-useful to those planning for the use or provisioning of data or maps - to highly technical pieces clearly targeted at those who might be designing the delivery systems. Some entries focus on data processing and data mining, while others take data acquisition as an unproblematic given, and instead describe public-facing interfaces or resulting impacts on map production. Parts II and III are well curated, with all chapters relating strongly to the theme, but Part I is less organized. Some of the chapters in Part I are clearly related to the theme-an exploration of this "new paradigm"-but others are singular use cases detailing the development of particular map services, some more successful than others. For example, one case study provides considerable detail on the planning and implementation of 
a DaaS framework to serve the needs of agencies across Europe. The study discusses the engagement of key stakeholders, the collaborative design of service components, and the development timeline-which has been lengthy and is still ongoing. Another example outlines a map creation tool for Catalonia, in northeastern Spain, that was developed in a top-down manner, with no user input, and that was determined to be too complex to use once implemented. Yet a third case study describes the development of an automated process to add pie charts to any type of flow map, resulting in some pretty egregious violations of cartographic principles.

As a whole, Service-Oriented Mapping is plagued with minor editorial concerns, and a few chapters are both very poorly edited and tenuously placed in the literature. In particular, the opening chapter of Part II, "Developing a Statistical Geospatial Framework for the European Statistical System" (185-206), discusses how census point data were used to develop the framework, yet never mentions pertinent issues surrounding the protection of personally identifiable information. Other chapters refer extensively to European programs by their acronyms, often without explanation, which may leave the whole discussion confusing to non-European audiences.

The graphics in the print version of this book are uniformly poor-with most quite grainy and otherwise unreadable-something that is quite disturbing in a book of this price. Some graphics are slightly better than others due to their overall simplicity, and might have been perfectly fine had they been submitted in black and white, but most are completely illegible due to font size or complexity, exacerbated by the printing method. Curious to see what the colored mess purporting to be a section of a nineteenth-century geologic map of Austria (346) actually looked like, I combed the Internet for a digital image, and found a sample from the eBook version of Service-Oriented Mapping. The digital version reveals the map in full glory, but the print version looks like it was produced on a lowend inkjet printer on the draft setting. Issues of print quality aside, many of the graphics suffer from microscopic text and unclear organization, and even in the eBook they remain incomprehensible. Chapter 19, "Supply Chains and Decentralized Map Productions" (385-416), relies on a number of flow charts and diagrams to "explain" its points, but with no descriptive narrative in the text, and no legend to explain the abbreviations used in the diagrams, the reader is left quite perplexed. Even aside from this chapter, so many of the so-called mapping techniques presented throughout this book are so focused on expediency at the expense of sound cartographic principles that cartographers may likely find the book of dubious value

There are a few chapters in here which made the book worth reading - although not, in my opinion, enough to merit the cost. In particular, some of the case examples were quite good; making note of relevant sticking points in the process, or elements that required particular care. Chapter 3, "Establishing Common Ground Through INSPIRE: The Legally-Driven European Spatial Data Infrastructure" (63-84) provided a nice review of an existing framework. The chapter describes the project's goals, components and stakeholders, while noting that the development process has taken longer than anticipatedtenyears to date, and still not at full implementationan important caution for agencies looking to undertake large-scale initiatives. Some of the discussions highlighted salient points about communication difficulties and standards disparities between agencies and between nations, and, similarly, some of the technical pieces described their methods with sufficient detail to make them entirely reproducible. Especially noteworthy is the contribution on and by the Spanish organization Vizzuality, creator of mapping applications for non-governmental organizations (NGOs). It provided three easy-to-digest case studies that include sufficient information to allow NGOs to understand the full benefit of making massive amounts of data available to, and manageable by, users ("Vizzuality: Designing Purposeful Maps to Achieve SDGs,” 207224). Also of note are Chapters 12, 15, and 16-all related to spatial data infrastructure. Chapter 12, "SDI Evolution and Map Production" (241-250) is on how such an infrastructure feeds map production, while the other two, "How Standards Help the Geospatial Industry Keep Pace with Advancing Technology" (303-324), and "StandardsMaking Geographic Information Discoverable, Accessible and Usable for Modern Cartography" (325-344), are about the need for standards, and all three are both technical and accessible to a wide audience. This last chapter is available separately from the publisher, along with three other less useful chapters, priced at $\$ 29.95$ each.

In general, however, Service-Oriented Mapping: Changing Paradigm in Map Production and Geoinformation Management is not sufficiently homogeneous to render it uniformly useful, and the issues with quality and consistency made it a difficult task to take in, or recommend, the entire book. 